\title{
PATRONES DE ASENTAMIENTO EN EL PERÍODO ALFARERO TEMPRANO DE CHILE CENTRAL
}

\author{
SETTLEMENT PATTERNS IN THE EARLY CERAMIC \\ PERIOD OF CENTRAL CHILE
}

\author{
Lorena Sanhueza R. ${ }^{1}$, Luis Cornejo B. ${ }^{2}$ y Fernanda Falabella $G .{ }^{1}$
}

\begin{abstract}
En este trabajo se presentan los resultados de un estudio sobre el patrón de asentamiento de los grupos del período Alfarero Temprano de Chile central. El análisis se llevó a cabo a partir de datos recopilados por medio de prospecciones sistemáticas realizadas en la cuenca de Rancagua, cuenca de Santiago y valles de Melipilla. Los resultados muestran un claro patrón de agrupamiento de los sitios en torno a cursos de agua, aunque hay espacios que aparentemente cumplen con las mismas condiciones, pero no están siendo utilizados. Se utilizan antecedentes etnográficos y etnohistóricos para proponer que este patrón de asentamiento responde a una organización social basada en la comunidad formada por conjuntos de familias con estrechas relaciones de cooperación entre sí y cuya economía de subsistencia está basada en la horticultura.

Palabras claves: Chile central, período alfarero temprano, patrón de asentamiento, sociedades hortícolas.
\end{abstract}

Essential to understanding prehispanic settlement systems is the identification of the structure and relationships of archaeological sites within a region. Here we examine the results of a survey program that included the Rancagua basin, Santiago basin and Melipilla valley in Central Chile, in order to make inferences related to the settlement pattern of groups that inhabited this area during the Early Ceramic Period. The data show clusters of sites grouped near water resources, mainly river valleys, and empty spaces that share the same geographical conditions but have no evidence of ancient occupation. We use ethnographic and ethnohistorical evidence to propose that this pattern relates to the social organization of groups, with families as the closest cooperative level, and whose subsistence economy is mainly horticulture.

Key words: Central Chile, Early Ceramic Period, settlement pattern, horticultural societies.

El período Alfarero Temprano en Chile central (PAT) tiene una larga historia de investigación que se remonta a la década de los sesenta, con los pioneros estudios de Berdichewsky (1964) y Silva (1964) en la costa, y que se consolidó luego en la década de los ochenta con los estudios de Falabella y Planella $(1979,1991)$ y Planella y Falabella (1987), que permitieron definir las principales unidades arqueológicas que se encuentran vigentes hasta hoy (Llolleo y Bato). En los últimos 15 años los estudios se han concentrado en el interior, donde se ha avanzado especialmente en afinar la definición de estas unidades, su distribución espacial y temporal, así como en la organización social de estos grupos, tecnología de producción cerámica y dieta (Falabella et al. 19951996; Sanhueza et al. 2003; Sanhueza 2004; Sanhueza y Falabella 2006; Falabella et al. 2007).

No se han realizado hasta ahora, sin embargo, estudios que aborden de manera sistemática el pa- trón y el sistema de asentamiento de estos grupos, aunque el tema se ha abordado de manera indirecta y parcial. Es así como se mencionan el asentamiento preferentemente en lomajes y terrazas asociadas a cursos de agua en el litoral y terrazas fluviales muy próximas a los cursos de agua, especialmente las rinconadas, en el interior, donde se destaca, al menos para el caso Llolleo, el hecho de que los asentamientos se ordenan a lo largo de los ríos siguiendo un patrón disperso (Falabella y Stehberg 1989).

El objetivo de este trabajo es presentar los patrones de asentamiento en el PAT a partir de una muestra de sitios obtenida de manera sistemática por medio de una prospección regional del valle del río Maipo y de las cuencas de Santiago y Rancagua, y discutir estos resultados en función de los modelos sociales que estamos manejando para estas sociedades (Falabella y Sanhueza 2005-2006; Sanhueza y Falabella 2006).

1 Departamento de Antropología, Universidad de Chile, Ignacio Carrera Pinto 1045, Nuñoa, Santiago, loresan@vtr.net; ffala@entelchile.net

2 Museo Chileno de Arte Precolombino, Bandera 361, Santiago, lcornejo@ museoprecolombino.cl 
Este período comprende un amplio lapso de tiempo. Sus inicios están marcados operativamente por la presencia de alfarería, tecnología que se consolida hacia el 200 a.C. y termina hacia el 1.000 d.C. Un primer momento, hasta el 200 d.C., se caracteriza por unidades sociales relativamente independientes, definidas como Comunidades Alfareras Iniciales. Estas son bastante diferenciadas entre sí, algunas presentan cultivos incipientes como la quínoa (Chenopodium quinoa) y a nivel de la alfarería comparten ciertos patrones comunes (Sanhueza y Falabella 1999-2000). Hacia el 200 d.C. se distinguen dos unidades contemporáneas, Bato y Llolleo, con diferencias explícitas en adornos corporales visibles, prácticas funerarias, estilos alfareros e implementos que utilizan. Sus patrones de subsistencia y asentamiento también difieren, más móviles y dependientes de recursos silvestres los primeros y más sedentarios y dependientes de los cultivos los segundos (Sanhueza et al. 2003). Ambas comunidades se consideran sociedades simples sin jerarquías formales y se interdigitan espacialmente en la zona septentrional de nuestra área de estudio. En la cuenca de Rancagua, donde la presencia del componente Llolleo es muy fuerte, no parecen haber evidencias del componente Bato, aunque sí de otros grupos, con características distintas a las anteriormente mencionadas.

Si bien existen estos distingos sociales y culturales, las evidencias arqueológicas señalan que los grupos del PAT son todos hortícolas. Los registros han mostrado que la quínoa (Chenopodium quinoa) está presente en contextos del período Arcaico IV, luego aparece entre los desechos de las Comunidades Alfareras Iniciales y hacia el 500 d.C., las poblaciones de esta zona manejan una variedad de cultivos además de la quínoa, entre los que se incluyen maíz (Zea mays), zapallo ( $\mathrm{Cu}$ curbita sp.) y poroto (Phaseolus vulgaris) (Planella y Tagle 1998, 2004; Quiroz y Belmar 2004). Los análisis de isótopos estables muestran que si bien hay variación intergrupal (Bato v/s Llolleo, costa/interior), estos dos grupos, especialmente en los valles del interior, están consumiendo una cantidad importante de maíz (Falabella et al. 2007).

Chile central es un área de características climáticas mediterráneas y con un relieve marcado por la definición de planicies costeras, la cordillera de la Costa, la depresión central y la cordillera de los Andes, en todos los cuales se han formado planos y terrazas con suelos aptos para la horti- cultura. Para los fines de este trabajo, interesa destacar que no se están considerando ciertos espacios que pueden haber sido parte del sistema de asentamiento y subsistencia de las poblaciones (costa y cordillera) y que las condiciones de paisaje, recursos y clima dentro del área de estudio son bastante similares (Quintanilla 1983).

\section{Patrones de Asentamiento y Sociedades Hortícolas}

Entendemos por patrón de asentamiento la estructura formal de los asentamientos en el espacio y por sistema de asentamiento las reglas de la sociedad que rigen esta ordenación (Chang 1968). El sistema de asentamiento es un reflejo de las actividades sociales y económicas y se estructura de acuerdo a la organización de las relaciones sociales. Los patrones de asentamiento son el resultado de la forma en que los grupos humanos se disponen en el espacio en función tanto de las características propias del medio ambiente que están ocupando (características físicas y ambientales), como de la tecnología que maneja el grupo para lograr su subsistencia a partir de éste (recolección, caza, producción de alimentos, irrigación, almacenamiento), combinado con la manera en que éstos se organizan socialmente para ello (organización social de la producción), ecuación que no es única ni estable.

Los modelos adaptacionistas privilegian las características del medio ambiente y los recursos (Winterhalder 1981). Nuestra postura es que existe una fuerte interrelación entre la localización de los asentamientos, la disponibilidad, distribución y calendario de uso de los recursos, la distribución de los distintos segmentos sociales o arreglos demográficos y la tecnología que maneja cada sociedad (Jochim 1976). En este sentido los patrones de asentamiento de los grupos del PAT debieran ser coherentes con el tipo de relaciones sociales y espaciales que pueden darse entre sociedades de baja escala con patrones de subsistencia hortícolas.

El concepto de sociedad "horticultora" como tipo económico es de difícil definición porque los límites con los recolectores y con los agricultores son difusos (Johnson 1989). Por cierto, el concepto implica el manejo de plantas domesticadas y una subsistencia parcialmente basada en cultígenos. Acorde con esto, se asume como una práctica 
a baja escala y con un manejo tecnológico restringido, donde son característicos los cultivos de tala y roza, el desplazamiento periódico de los campos por el agotamiento de los suelos y la ausencia de tecnologías de mejoramiento de éstos. Los huertos tienden además a ser de policultivos, reuniendo una variedad de especies en un mismo espacio, incluso a veces de manera interdigitada. Sin duda esto se correlaciona hasta cierto punto con la escala de la unidad de producción, que es la familia o el grupo de parentesco, que produce principalmente para la subsistencia.

En este sistema de producción a baja escala y con tecnología poco especializada, características como la humedad de los suelos, pasa a ser relevante, especialmente en climas mediterráneos como el de Chile central, donde es esperable que los campos de cultivos, y por ende los asentamientos, se dispongan en terrenos con napas freáticas altas, o bien próximos a los ríos con las condiciones de humedad necesarias para el adecuado desarrollo de los cultivos, sin el desarrollo de sistemas complejos de irrigación. Al respecto, hay que considerar las diferencias que presentan las distintas especies cultivadas en relación a sus requerimientos. En el caso de los cultivos encontrados en Chile central, la quínoa es un seudocereal muy adaptable y resistente, pero el maíz presenta mayores exigencias en agua y minerales.

Una de las características que muchas veces se asocian a la horticultura es el sedentarismo. Esta idea surge del contraste entre la movilidad de cazadores recolectores que se mueven varias veces al año en función de la obtención de los recursos y de la relación de los horticultores con sus campos de cultivo, que exige la presencia directa de los grupos (o al menos de parte de ellos) en las etapas de preparación de los huertos, la siembra y la cosecha, y también por la necesidad de cuidado y protección de los cultivos, que en un clima con una estación seca marcada y prolongada puede implicar ciertas prácticas de irrigación.

Algunos estudios, sin embargo, han mostrado que este tipo de sociedades también presenta patrones de movilidad, solo que es una movilidad diferente, por lo general referida al traslado de las viviendas cada una o dos generaciones y determinados principalmente por la rotación de los campos de cultivo y por necesidades sociales (Dillehay 1992; Johnson y Earle 1987; Varien 1999). "Sedentarismo" es, entonces, un concepto que in- cluye en su definición una gama de situaciones diferentes y puede referirse también a grupos con una baja frecuencia de movimiento del lugar de residencia (Chatters 1987; Rafferty 1985).

La mayor permanencia en un territorio más restringido, por otra parte, lleva a estas sociedades a organizar sus asentamientos de forma tal que sea posible la interacción necesaria con otros para la cooperación económica, el flujo de información y la reproducción social. El espaciamiento entre viviendas y las distancias deben ser compatibles con estas necesidades sociales. Esperaríamos encontrar agrupamientos correspondientes a unidades sociales interactuantes y no sitios dispersos y aislados en el paisaje. En este marco, es esperable además que este ordenamiento refleje los distintos niveles de relaciones sociales que hemos propuesto para las sociedades del PAT, tales como grupo corresidencial, comunidades y unidades socioterritoriales (Falabella y Sanhueza 2005-2006).

\section{La Prospección Regional}

Este estudio se ha realizado a nivel regional comprendiendo un amplio espacio de $4.062 \mathrm{~km}^{2}$, que incluye el área de distribución de los complejos culturales ya mencionados y que hemos propuesto como la escala más amplia de integración social (Falabella y Sanhueza 2005-2006). No ha sido posible incluir, en este análisis, los asentamientos costeros que hacen parte de los grupos sociales estudiados por no disponer de prospecciones equivalentes a las del interior. Esperamos entonces distinguir distintas escalas espaciales con correspondencia en unidades sociales tales como la unidad socioterritorial, la comunidad y el grupo corresidencial.

Los datos utilizados en este trabajo provienen de una prospección sistemática realizada en las cuencas de Santiago y Rancagua, así como en los valles de Melipilla. Esta base de datos tiene beneficios para el problema planteado, pero también conlleva algunas importantes limitaciones. Obviamente, un estudio sobre el patrón de asentamiento requiere conocer la forma en que se distribuyen en el espacio una muestra representativa de asentamientos de la población o período en cuestión. Normalmente, tal como en nuestro caso, esta muestra contendrá una gran proporción de sitios caracterizados principalmente a partir de la superficie, 
lo que posibilita registrar un gran número de ellos, sin una inversión demasiado grande de recursos. Sin embrago, presenta el inconveniente de ocultar parte de la diversidad interna de los sitios, la cual en algunos casos puede ser significativa cultural y socialmente. Así, se sacrifica detalle por amplitud de la muestra.

En nuestro caso de estudio, el PAT, la diversidad interna hace referencia a conjuntos culturales que se diferencian sustancialmente, pero cuyas expresiones materiales son de un nivel de sutileza que generalmente requiere de excavaciones extensivas para ser identificados (Sanhueza et al. 2003). De esta manera, nuestra base de datos pone énfasis en características que permiten diferenciar los sitios en términos de la macroestructura histórico cultural de la región, diferenciando sitios del PAT de los del período Intermedio Tardío y Tardío, pero no permitiendo identificar claramente a cuál de los tres distintos complejos culturales que se han identificado en esta área (Llolleo, Bato o Comunidades Iniciales) pertenece cada asentamiento o, eventualmente, si estamos enfrentándonos con otros componentes culturales no identificados previamente. Por otra parte, tampoco estamos controlando con finura la cronología al interior de este largo período que ocupa más de 1.000 años de la secuencia regional, y al interior del cual muy probablemente ocurrieron cambios, asociados a la consolidación del nuevo sistema de subsistencia relacionado con la producción de alimentos.

Pese a estas limitaciones, que nos permite referirnos sólo a las ocupaciones del PAT en términos generales, creemos que es un avance tener la imagen de cómo se ocupó el espacio durante este período, resultados que son sustanciales para comenzar a comprender las dinámicas sociales de estas poblaciones. A la vez, los resultados que aquí presentamos tienen una estructura que parece indicarnos la existencia de rasgos del patrón de asentamiento que son generalizables a todos los grupos. Por lo demás, para intentar descartar que los resultados sean un artefacto de la muestra, también los compararemos con la distribución de sitios del período Alfarero Temprano conocidos a raíz de otras investigaciones y Estudios de Impacto Ambiental.

Nuestra base de datos proviene de una prospección diseñada a partir de un Muestreo Probabilístico Sistemático No Alineado. El universo de estudio estuvo formado por las tierras planas de las tres áreas prospectadas (valles de Melipilla, cuenca de Rancagua y cuenca de Santiago), con un límite en el comienzo de las pendientes de los cerros que las rodean (pendiente $+/-5 \%$ ). En él se aplicó una estrategia de muestreo ya utilizada previamente (Cornejo et al. 2003-2004), especialmente adaptada a los problemas de alta subdivisión de la propiedad de la tierra y el uso agrícola de la tierra que caracterizan este territorio. De esta manera, nuestra prospección debería evaluarse en función de problemas de accesibilidad (propiedad de la tierra y actividades agrícolas) y visibilidad (actividades agrícolas), un muestreo probabilístico determinado y una intensidad de $100 \mathrm{~m}$. Si bien estamos trabajando en un área fuertemente impactada por procesos postdepositacionales derivados de la agricultura, ganadería y la industrialización de los últimos siglos, éstos han afectado a toda la región, por lo que no constituye un factor de sesgo. Los sitios fueron delimitados con una franja de 100 metros de espacio culturalmente estéril en superficie y no fueron diferenciados por sus tamaños, ya que la mayor parte de ellos no están delimitados por todos sus flancos. Por otra parte, la realización de pozos de sondeo ha mostrado que la distribución superficial de los materiales generalmente es mucho más amplia que su distribución en estratigrafía.

Un último factor para sopesar esta prospección es la fracción de la muestra. En conjunto, el territorio estudiado define un universo de $4.062 \mathrm{~km}^{2}$, de los cuales se prospectaron 167,6 $\mathrm{km}^{2}$, lo que equivale a una fracción de $4,1 \%$. Sin embargo, en cada una de las tres áreas prospectadas no se prospectó la misma fracción (Tabla 1). Estas diferencias se deben básicamente a los costos asociados y al tamaño de las áreas comprometidas. Obviamente, la fracción de las muestras estudiadas, especialmente en Santiago y Melipilla, son pequeñas, por lo cual seguramente la prospección no ha sido capaz de identificar los detalles más finos de la distribución del asentamiento y tampoco ha podido localizar toda la diversidad posible de sitios arqueológicos. No obstante, estas limitaciones se ven aminoradas por el hecho que nuestros principales resultados derivan de la comparación entre territorios prospectados con la misma intensidad, lo cual permite construir hipótesis con cierto grado de confiabilidad. 


\section{Resultados}

El balance de los resultados de la prospección y de las inferencias sobre el patrón de asentamiento que de ella se pueden extraer será abordado en primer lugar desde la perspectiva de cada una de las tres regiones estudiadas, para luego proponer una visión de conjunto.

\section{Cuenca de Rancagua}

Esta cuenca es la que ha sido prospectada en una fracción mayor $(8,5 \%)$, razón por la que los patrones que hemos detectado en la distribución de los asentamientos del PAT pueden ser postulados con una certeza razonable. Esta prospección produjo un total de 60 sitios PAT, los que no se distribuyen homogéneamente. Existen áreas donde se concentran los asentamientos, mientras que en otras están prácticamente ausentes, pese a que se invirtió la misma intensidad y fracción de la muestra (Tabla 1). En la Figura 1 se pueden observar las unidades de prospección en las cuales se localizaron sitios y aquellas donde no se produjeron hallazgos, lo cual define con precisión localidades diferentes.

Junto con lo anterior, al considerar los aspectos fisiogeográficos básicos de esta cuenca, se hace evidente que los asentamientos se concentran en torno a los principales cursos de agua de ésta, mientras que en las áreas donde nos hay esteros o ríos importantes simplemente no hay asentamientos. Así, es posible definir cinco espacios relativamente independientes. Las áreas que concentran los asentamientos definen dos espacios relativamente pequeños en el Norte, uno en torno al estero Peuco y otro en torno al estero Codegua, en el sur un área de tamaño medio en torno al río Claro y una región muy amplia en el centro norte, en torno al estero La Cadena y el Río Cachapoal. Esta última localidad eventualmente puede subdividirse en dos, ya que existe un territorio sin asentamientos que divide los ubicados al norte y al sur de Lo Miranda. Considerando ambas vertientes del río Cachapoal en este espacio se prospectó cerca de $5 \mathrm{~km}^{2}$ sin localizar ningún sitio, mientras que la media en las áreas con asentamientos es cercana a $1 \mathrm{sitio} / \mathrm{km}^{2}$.

Por su parte, la ausencia de asentamientos es evidente en cuatro áreas. Una en el norte, entre los esteros Codegua y Peuco, por el cual escurre el pequeño estero Picarquín. En el sur se localiza una pequeña localidad, que está directamente asociada a las riberas del río Cachapoal antes de la junta con el río Claro. En el norte se define un

Tabla 1. Localidades estudiadas. Studied locations.

\begin{tabular}{|c|c|c|c|c|c|}
\hline & $\begin{array}{c}\text { Superficie } \\
\text { Prospectada }\left(\mathrm{km}^{2}\right)\end{array}$ & $N^{\circ}$ Sitios & $\begin{array}{l}\text { Densidad Sitios/ } \\
\text { Sup. Prospectada }\end{array}$ & $\begin{array}{l}\text { Distancia } \\
\text { Mínima } \\
\text { al Vecino } \\
\text { más Cercano }\end{array}$ & $\begin{array}{r}s \text { Distancia } \\
\text { Mínima } \\
\text { al Vecino } \\
\text { más Cercano }\end{array}$ \\
\hline RANCAGUA & & & $1.200 \mathrm{~km}^{2}$ & & \\
\hline Cachapoal-Cadena & 21,8 & 20 & 0,9 & 2,04 & 1,72 \\
\hline Claro & 20,3 & 22 & 1,1 & 1,46 & 0,87 \\
\hline Codegua & 4,5 & 6 & 1,3 & 2,7 & 0,79 \\
\hline Peuco & 3 & 8 & 2,7 & 0,40 & 2,88 \\
\hline Total & $101,7(8,5 \%)$ & & & & \\
\hline SANTIAGO & & & $2.402 \mathrm{~km}^{2}$ & & \\
\hline Angostura & 4,5 & 15 & 3,3 & 0,98 & 0,62 \\
\hline Buin-Maipo & 4,6 & 4 & 0,9 & 1,09 & 0,78 \\
\hline Colina & 2,1 & 3 & 1,4 & 3,1 & 3,63 \\
\hline Maipo-Mapocho & 7,4 & 10 & 1,4 & 2,25 & 2,25 \\
\hline Total & $39,4(1,6 \%)$ & & & & \\
\hline MELIPILLA & & & $460 \mathrm{~km}^{2}$ & & \\
\hline La Higuera & 3,23 & 5 & 1,5 & 0,86 & 0,58 \\
\hline Peralillo & 1,97 & 3 & 1,5 & 1,73 & 0,92 \\
\hline Maipo & 2,85 & 2 & 0,7 & $\mathrm{n} / \mathrm{a}$ & $\mathrm{n} / \mathrm{a}$ \\
\hline Total & $23,9(5,1 \%)$ & & & & \\
\hline
\end{tabular}


espacio que incluye parte de la cuenca este del estero La Cadena y los territorios circundantes hasta cerca del estero Codegua, por el norte, y el río Cachapoal por el suroeste. Por su parte en el centro sur de la cuenca, entre el río Cachapoal y el río Claro, se extiende una gran área donde sólo se localizó un sitio PAT, y que se caracteriza por no estar irrigada por ningún curso de agua permanente, con excepción de su segmento sureste, donde escurre el estero Tipaume, afluente del río Claro.

Una parte importante de estas áreas "no ocupadas" en el PAT se pueden caracterizar por su deficiencia de recursos hídricos. No obstante esta característica sólo sirve para explicar de manera parcial la ausencia de asentamientos, ya que parte de ellos sí tiene cursos de agua, tales como el estero Picarquín en el norte, la parte superior del estero La Cadena en el centro norte o el estero Tipaume en el sureste. Por su parte, la ausencia de sitios en el extremo sur del río Cachapoal podría explicarse por tratarse la mayor parte de esa localidad de áreas de inundación de este río.

\section{Cuenca de Santiago}

Este territorio fue el que se prospectó en una fracción menor (1,6\%), lo que se condice con su gran extensión, que involucra $2.402 \mathrm{~km}^{2}$, y el fuerte impacto urbanístico del área. En ésta se localizó un total de 34 asentamientos PAT. Los parámetros básicos de la prospección realizada en Santiago (Tabla 1) nos indican que pese a la importante diferencia en la fracción de la muestra prospectada entre esta cuenca y la de Rancagua, no existen diferencias sustanciales en la frecuencia con que se presentan los asentamientos PAT en estas dos áreas. Al observar cómo estos asentamientos se distribuyen en el espacio se reconocen otras similitudes, así como también importantes diferencias.

Por un lado, y a diferencia de lo que ocurre en Rancagua, los sitios reconocidos en la prospección en Santiago se concentran únicamente en los extremos norte y sur de esta cuenca, existiendo un extenso territorio sin hallazgos (Figura 1). Una parte importante de estas áreas sin asentamientos podría ser explicada por problemas de visibilidad derivados de una alta sedimentación en terrenos de inundación o de napas freáticas muy superficiales, especialmente en las locali- dades en torno a Lampa y el estero Colina en el norte, pero también por fracciones muy bajas de prospección, especialmente en el río Clarillo. No obstante, estos criterios no pueden aplicarse a los territorios prospectados en torno al río Mapocho o los que su ubican al sureste del río Maipo.

Por otro lado, es evidente que al igual que en Rancagua, la distribución de los asentamientos parece estar vinculada principalmente a los cursos de agua más importantes de la cuenca. Del mismo modo, se pueden identificar localidades donde parecen concentrarse los asentamientos, identificándose claramente tres de ellas. En el norte de la cuenca se define la localidad de Colina, la cual presenta una densidad muy baja de sitios. En el sur de la cuenca, donde se concentra la mayor parte de los asentamientos identificados, se reconoce un área muy densa de asentamientos en torno al río Angostura y sus afluentes, así como otra similar en el territorio intermedio y cercano a la confluencia de los ríos Maipo y Mapocho (Tabla 1). Entre ambas se extiende la localidad de Buin-Maipo, donde la densidad de sitios es baja. En cada una de estas dos localidades densamente ocupadas, los asentamientos se encuentran más agrupados que en ninguna de las regiones aquí estudiadas. La distancia media al vecino más cercano en el río Angostura es de 0,98 km $(s=0,62)$, mientras que en la localidad MaipoMapocho es de $1,09 \mathrm{~km}(s=0,78)$, lo cual resulta significativamente menor que lo ya detallado en la cuenca de Rancagua (Tabla 1). Esto es especialmente significativo considerando que la fracción de la muestra prospectada en cada una de estas dos cuencas es muy distinta, por lo que sería esperable que en Rancagua, donde se prospectó una fracción mucho mayor que en Santiago, la distancia promedio entre los sitios fuera menor. Del mismo modo, resulta interesante consignar que en estas dos localidades se verifica una densidad de sitios localizados por cada kilómetro prospectado mayor que en las localidades estudiadas en Rancagua (Tabla 1), destacando especialmente la densidad en torno al río Angostura, la cual prácticamente triplica la densidad de sitios que la presente en la mayor parte de las localidades de Rancagua.

En este punto es interesante destacar que en los dos últimos parámetros en que hemos comparado la distribución del asentamiento entre las 


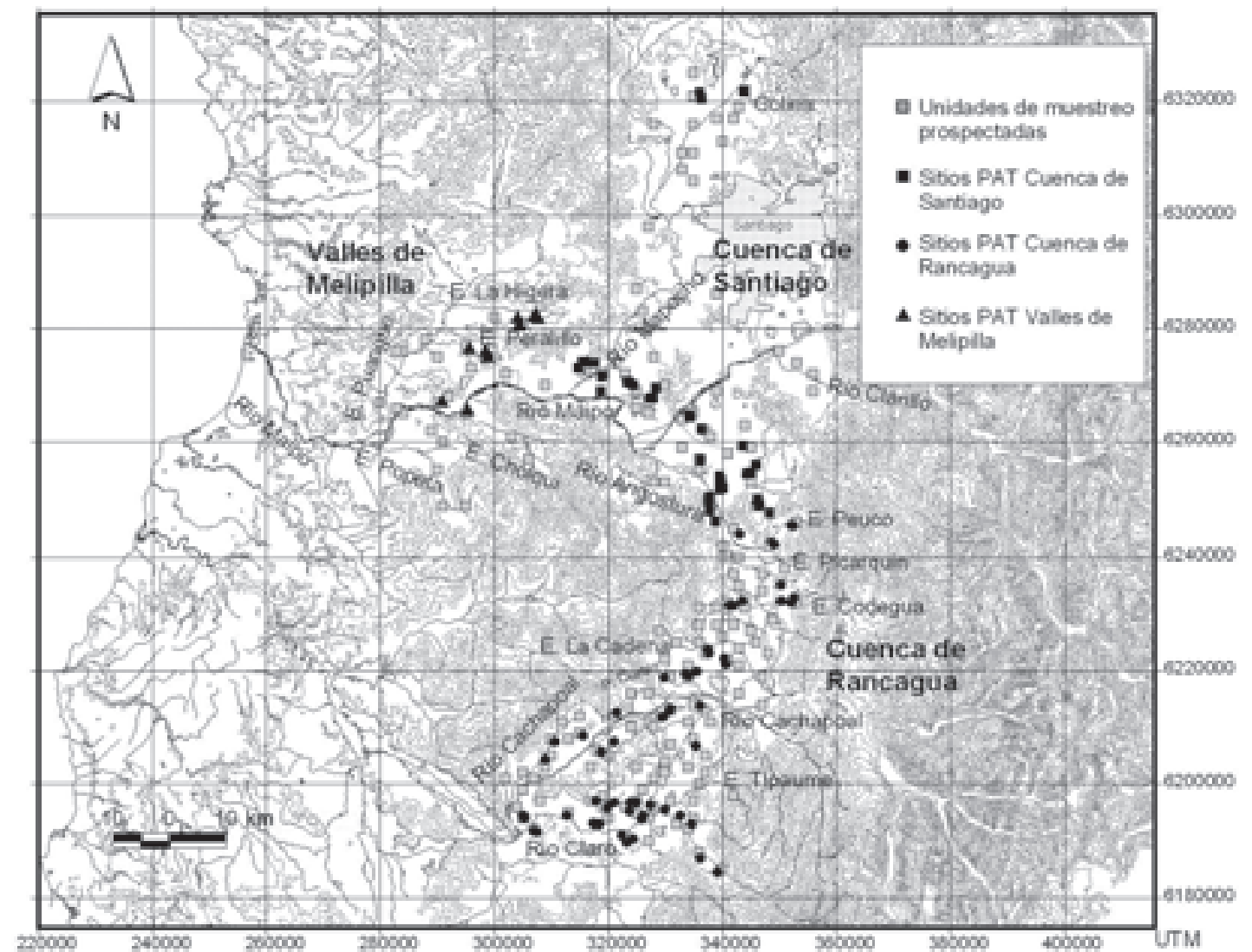

Figura 1. Distribución de sitios PAT reconocidos en la prospección del área de estudio.

Distribution of Early Ceramic Period sites identified in the regional survey.

cuencas referidas, las localidades del río Angostura (Santiago) y estero Peuco (Rancagua) son muy similares. En ambas se dan las más altas densidades de sitios descubiertos por cada kilómetro cuadrado prospectado y la distancia media menor al vecino más cercano (Tabla 1). Esto seguramente se debe a que la localidad del estero Peuco realmente corresponde a la cuenca del río Angostura y que la separación que hemos hecho de ambas cuencas (Santiago y Rancagua), por la cadena de cerros que cierra la Angostura, realmente no debe ser considerada como un límite válido en términos culturales. Es probable entonces que los sitios del estero Peuco deban ser afiliados a la localidad del río Angostura.

\section{Valles de Melipilla}

Esta localidad es la más pequeña de las tres estudiadas y, a su vez, se diferencia por no corresponder a un gran valle aluvial como las cuencas de
Santiago y Rancagua, sino que precisamente por tratarse de un conjunto de valles que desde la cordillera de la Costa desembocan en el río Maipo. Estas características, en principio, podrían ser los agentes que definen la gran diferencia que presenta el patrón de asentamiento PAT aquí registrado en comparación con las otras dos regiones estudiadas.

En primer lugar, la densidad de 0,41 sitios por $\mathrm{km}^{2}$ prospectado pone a estos valles como los menos densos en asentamientos PAT de Chile Central. A la vez, al observar la Figura 1 se puede concluir que la distribución de los asentamientos es la menos homogénea de las tres regiones aquí estudiadas. De hecho, nueve de los diez sitios aquí registrados se localizan al norte de río Maipo, especialmente en dos afluentes del estero Puange, dejando los valles del sur (Cholqui y Popeta) prácticamente no ocupados por asentamientos PAT.

A la vez, en el territorio norte donde se encuentran los asentamientos PAT es posible identificar dos localidades donde se agrupan los sitios, 


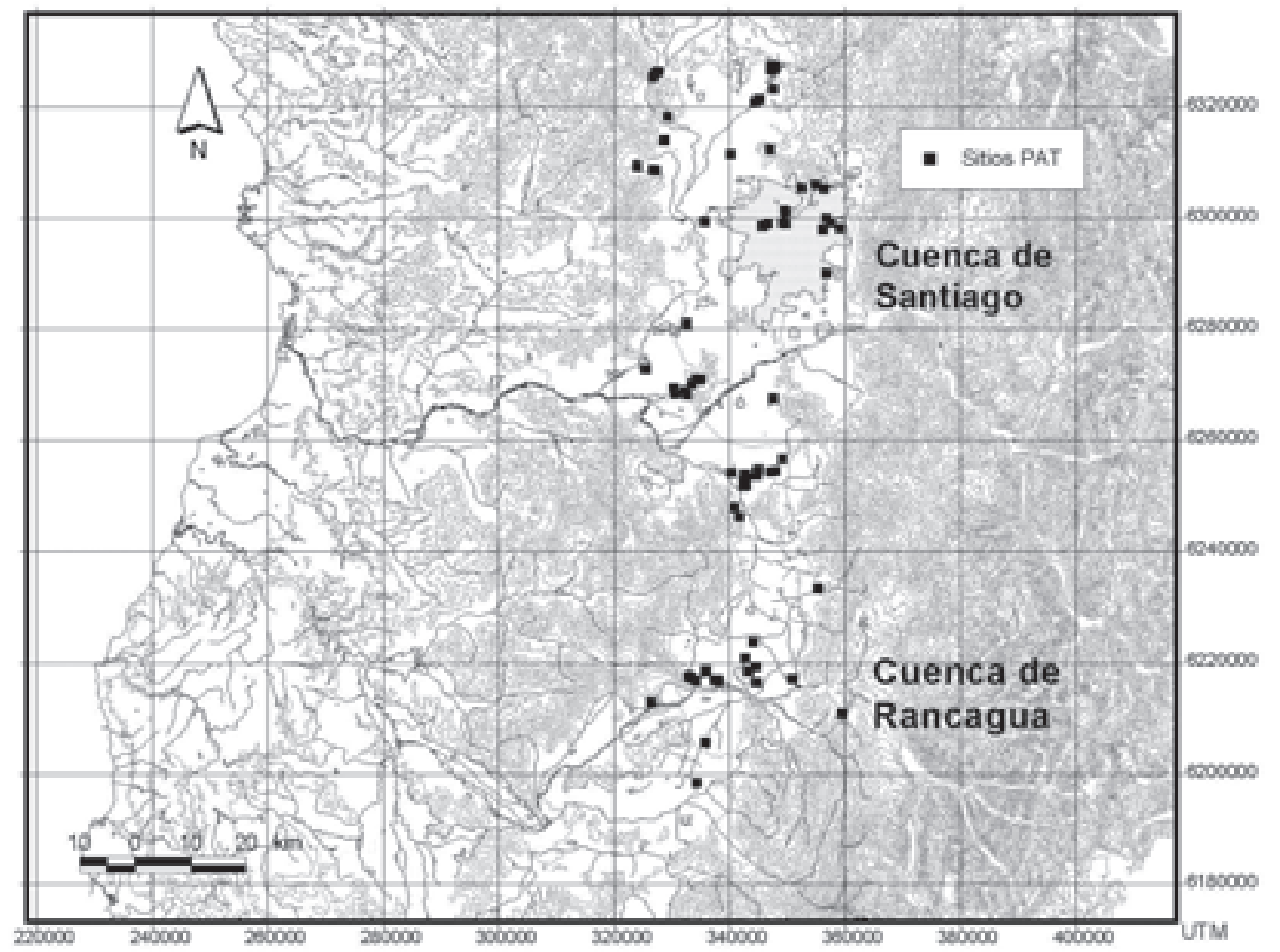

Figura 2. Distribución de sitios PAT reconocidos por otras investigaciones y Estudios de Impacto Ambiental en el área de estudio.

Distribution of Early Ceramic Period sites recognized in other surveys and Cultural Research Management studies from the area.

una en el estero La Higuera y el otro en la parte superior del estero Peralillo. Por su parte, dos sitios se encuentran dispersos cerca de las riberas del río Maipo, uno de los cuales es el único que se identificó al sur de este cauce. Las dos localidades con asentamientos, vistas desde el punto de vista de la densidad de sitios particular y la distancia mínima al vecino más cercano, sin embargo, no son muy distintas a otras localidades ya estudiadas en las cuencas de Santiago y Rancagua (Tabla 1), caracterizándose por una concentración de los asentamientos en áreas relativamente delimitadas y separadas de otros por áreas sin ocupación.

\section{Contrastación}

Obviamente, un problema importante en estos hallazgos es verificar si el comportamiento descrito puede ser un artefacto de la muestra, es- pecialmente considerando que el tamaño de ella no es similar en todas las regiones analizadas. En nuestro caso, la utilización de un esquema de muestreo probabilístico, tiende a disminuir esta fuente de error. No obstante, y para disminuir la incerteza, hemos contrastado nuestros resultados con una muestra proveniente de una base de datos elaborada a partir de datos generados por diversas investigaciones anteriores y Estudios de Impacto Ambiental, la cual, por los diversos orígenes de los datos, puede en su conjunto ser considerada determinada por el azar (Cáceres 1998; Cáceres 2003; Contreras 1999; Falabella 2000; Gaete 1998; Hagn 1998; Hermosilla 1998, 2001; Massone 1978; Ocampo 1999; Rees 2003; Reyes 2001, 2003; Salazar y Mera 1997; Sánchez 2001; Seelenfreund 1996, 1999a, 1999b, 2000; Stehberg 1976; Thomas et al. 1980, 1990; Westfall 1996, 2000, 2001, 2003). 
Esta contramuestra, compuesta por sitios PAT de funebria y habitacionales de las cuencas de Santiago y Rancagua, genera una distribución que en su conjunto fortalece las conclusiones propuestas por el estudio de los datos de nuestra prospección y, a la vez, los amplía (Figuras 1 y 2). Por un lado, es evidente que, al igual que los sitios de nuestra prospección, estos sitios se concentran en torno a los cursos de agua, formándose agrupaciones que en la mayor parte de los casos coinciden con las nuestras, especialmente en las localidades de Cachapoal-La Cadena (Rancagua), río Angostura (Santiago), Maipo-Mapocho (Santiago) y Colina (Santiago). A la vez, aparecen nuevas concentraciones en lugares por nosotros no prospectados. En la ciudad de Santiago, imposible de prospectar por la densidad urbana, se registra una importante cantidad de sitios que se concentra en las cercanías del río Mapocho y, especialmente, en torno al antiguo curso del estero San Ramón. Del mismo modo, en torno a la localidad de Lampa, prácticamente no muestreada por nosotros, aparece otra concentración de asentamientos.

\section{Discusión}

Las características de los sitios y la distribución espacial de los asentamientos del PAT permiten destacar algunos elementos centrales. En primer lugar, todos los sitios registrados en la prospección son abiertos y de tipo habitacional. En general las concentraciones de basura en los sitios excavados son de dimensiones reducidas (entre 500 y $1.000 \mathrm{~m}^{2}$ ) y de escasa potencia estratigráfica, lo que es coherente con una permanencia poco prolongada en cada lugar y el traslado recurrente de las viviendas. Debemos considerar que la densidad "real" de sitios que estuvieron funcionando en forma contemporánea es aún menor que la que se aprecia en las figuras. No sólo estamos juntando al menos unos 800 años, sino que distintos sitios pueden haber sido ocupados por el mismo grupo de personas.

Distintos factores pueden influir en ello. Por una parte, la posibilidad de agotamiento de los suelos. Cultivos como el maíz agotan rápidamente los suelos (especialmente el nitrógeno) y, al no disponer del uso de fertilizantes, conlleva la necesidad de rotación de los campos o del tipo de cultivos. Es muy sugerente el caso de grupos como los Machiguenga, quienes despejan cada año un huerto nuevo para plantar maíz aunque el suelo aún sea productivo y para quienes las tierras "fértiles" tienen características mucho más acotadas que para la apreciación occidental (Johnson 1989). En este sentido estamos conscientes que pueden existir percepciones culturales que evalúen de manera diferente el potencial del entorno de los asentamientos.

En segundo lugar, es evidente que los sitios no se distribuyen aleatoriamente en el espacio sino que forman agregados en torno a los principales cursos de agua, y sólo ocupan marginalmente los territorios alejados de éstos. El agrupamiento de sitios en el espacio puede deberse a diversos factores. Uno es la localización de las condiciones más óptimas para desarrollar su economía de subsistencia. La ubicación preferente cercana a los ríos es coherente con un patrón de subsistencia hortícola, que necesita suelos húmedos o fuentes de agua cercanas para regar los cultivos. En este sentido es notable la concentración de asentamientos en áreas con napas freáticas muy altas (p.ej. en la localidad del río Angostura), o cerca de los cursos de agua menores o esteros tributarios de los grandes ríos que cruzan el área (Maipo y Cachapoal), que deben haber sido más fáciles de manejar para la extracción de agua.

Asociado a esto está la existencia de "espacios vacíos" entre áreas que concentran el asentamiento, que, como hemos señalado, no siempre se relacionan con la inexistencia de cursos de agua, tal como en algunos esteros o parte del río Maipo en Melipilla, parte del río Mapocho en Santiago y parte del río Cachapoal y el estero La Cadena en Rancagua.

Una alternativa para explicar este patrón de agrupamiento/espacios vacíos, que dan la impresión de un paisaje subutilizado, puede encontrarse en la demografía regional, que nunca parece haber correspondido a una población muy numerosa (Téllez 2004). No obstante, la existencia de zonas con las mismas potencialidades de recursos que no fueron ocupadas, nos hace pensar que los recursos hídricos, ya sea en forma de cursos de agua o humedad generada por napas freáticas altas, pudo ser el motivo inicial de un asentamiento en el lugar, pero que la permanencia a través del tiempo y la ocupación de ciertos espacios y no de otros trasciende la disponibilidad y localización de éstos. De esta manera, y si bien no descartamos el factor demográfico como una varia- 
ble que también puede estar pesando, creemos que estos agrupamientos se materializan a partir de la forma en que los grupos PAT se organizan socialmente.

En este sentido proponemos que estas agrupaciones en torno a los ríos tienen un correlato social en familias extendidas relacionadas unas con otras por medio de lazos de parentesco o "unidades socioterritoriales" (Planella 1988). Los sitios representarían unidades corresidenciales de familias extendidas; en la medida en que esta unidad crece, a través de matrimonios y la reproducción, los grupos se fragmentan, generando nuevos asentamientos en las proximidades. Esta propuesta se basa ciertamente en situaciones etnográficas y etnohistóricas (Dillehay 1995; Falabella y Sanhueza 2005-2006), pero también encuentra su lógica en que los grupos de personas que viven en sitios próximos tienen una posibilidad real de interacción, que a medida que aumenta la distancia, se dificulta, especialmente en el marco de un patrón de movilidad menor.

Los "espacios vacíos", por su parte, sugieren la conformación de espacios limitados (en términos sociales más que medioambientales) que podrían aludir a una suerte de territorialidad. En este sentido las fronteras serían de índole social, en concordancia con las redes de parentesco y los linajes.

$\mathrm{Al}$ respecto, tomando en consideración las continuidades histórico culturales planteadas entre ambas áreas (Aldunate 1989), aunque guardando las distancias impuestas por la geografía y la cronología, nos parece sugerente la organización social y económica planteada en los últimos tiempos para los grupos que habitaban la zona sur a la llegada de los españoles. De Boccara (1998) destacamos los niveles mínimos de integración social para estos grupos (familia polígama, patrifamilia, quiñelob y lebo), que se traducen en un patrón de uso del espacio de caseríos dispersos, pero cercanos, cuyos miembros mantienen relaciones sociales y de cooperación económica entre sí. Bengoa (2003), por su parte, ha destacado que esta sociedad habría estado instalada y organizada en torno a los ríos, coincidiendo sus agrupaciones sociales con cuencas y espacios ribereños de lagos y mares, constituyendo de esta manera "sociedades ribereñas". Esto es tan evidente, que los españoles incluso nombran a los indígenas y los ríos con el mismo apelativo, situación que también ocurre en Chile central (p.ej. Cachapoal, Tinguiririca).
En nuestra propuesta no nos estamos olvidando de algo que ya señalamos al principio de este trabajo: nuestro nivel de resolución es el período, el que cubre un lapso temporal considerable y presenta una variabilidad interna que es significativa. Estamos conscientes, por tanto, de que al interior de estas agrupaciones debe existir una heterogeneidad cultural y ciertamente cronológica. No obstante lo anterior, la disposición de los asentamientos en el espacio es regular y recurrente, conformando en definitiva un verdadero patrón. Por lo tanto, parece evidente que las decisiones de donde asentarse son compartidas por los (eventuales) distintos grupos, aunque tengan distintos énfasis en la subsistencia dentro de un marco hortícola.

Estas diferencias, sin embargo, no alcanzan a ser tan importantes como las existentes entre estos grupos del PAT y los grupos Aconcagua de la cuenca de Santiago, que se caracterizan por ocupar no sólo otros espacios, sino con un espaciamiento diferente (Cornejo et al. 2003-2004). Esto refuerza lo que planteamos en relación a los factores que inciden en el análisis de los patrones de asentamiento y pone el acento en la necesidad de manejar las sutilezas, ya que muestra claramente cómo dos grupos que manejan cultivos similares y que se desarrollan en un mismo medioambiente usan el espacio de acuerdo a pautas diferentes, cuyas razones hay que buscar en factores socioculturales y organizacionales.

\section{Recapitulación: Hacia una Arqueología de la Localidad}

Como conclusión, este trabajo nos ha permitido obtener una visión clara de cómo se ordenan los asentamientos del PAT en Chile central. A la vez, y a partir de los modelos de organización social que estamos manejando y datos etnohistóricos del momento del contacto (siglos XVI y XVII), nos ha permitido generar ciertas propuestas relacionadas con la significación social de las agrupaciones identificadas. Estamos proponiendo que el patrón de asentamiento que hemos identificado para los sitios PAT en torno a determinados cursos de agua, obedece a una forma de organización basada en comunidades formadas por grupos de familias que interactúan estrechamente, lo que entendemos como una unidad "socioterritorial". 
En este marco, creemos que es importante comenzar a desarrollar una arqueología del PAT centrada en la localidad, que nos permita refrendar o rechazar estas propuestas, ya que la perspectiva que se nos abre es un correlato espacial claro a unidades sociales significativas al interior de las sociedades que estamos tratando: los grupos de parentesco básicos que permiten la reproducción de la sociedad (Falabella y Sanhueza 2005-2006). De esta manera podríamos abordar problemáticas relacionadas con la conformación de estas unidades y las relaciones entre ellas, que hasta ahora no han sido abordadas y que permitirán llegar a una mayor comprensión de los grupos estudiados.
Agradecimientos: Este trabajo es producto de las investigaciones realizadas en los proyectos FONDECYT 1970910, 1980713 y 1030667. Se agradece en forma especial a los ayudantes del proyecto FONDECYT 1030667: Itaci Correa, Elvira Latorre, César Miranda, Angela Peñaloza y Paulina Peralta. A los alumnos del curso "Métodos y Técnicas de Terreno" de la carrera de Arqueología de la Universidad de Chile de los años 2003,2004 y 2005 y a todos los que participaron de las prospecciones que permitieron generar la base de datos de los sitios utilizados en este trabajo. Se agradecen los comentarios realizados por los/las evaluadores/ras del manuscrito.

\section{Referencias Citadas}

Aldunate, $\mathrm{C}$.

1989 Estadio alfarero en el sur de Chile. En Culturas de Chile. Prehistoria, editado por J. Hidalgo, V. Schiappacasse, H. Niemeyer, C. Aldunate e I. Solimano, pp. 32948. Editorial Andrés Bello, Santiago.

Bengoa, J.

2003 Historia de los Antiguos Mapuches del Sur. Catalonia, Santiago.

Berdichewsky, B.

1964 Arqueología de la desembocadura del Aconcagua y zonas vecinas de la costa central de Chile. Actas del III Congreso Internacional de Arqueología Chilena, pp. 69104. Sociedad Chilena de Arqueología, Santiago.

Boccara, G.

1998 Guerre et Ethnogenese Mapuche dans le Chili Colonial. L'Invention du Soi. Traducido por D. Milos. L'Harmattan, France.

Cáceres, I.

1998 Informe Arqueológico. Prospección mediante pozos de sondeo en Larapinta, comuna de Lampa. Manuscrito en posesión del Consejo de Monumentos Nacionales.

2003 Excavación y rescate sitio arqueológico La Berlina, Comuna de Paine, Región Metropolitana. Manuscrito en posesión del Consejo de Monumentos Nacionales.

Chang, K.

1968 Toward the science of the prehistoric society. En Settlement Archaeology, editado por K. Chang, pp. 1-9. National Press, Palo Alto, California.

Chatters, J.C.

1987 Hunter-gatherer adaptations and assemblage structure. Journal of Anthropological Archaeology 6:336-75.

Contreras, L.

1999 Prospección y sondeos en el sector norte del Estero La Berlina. Proyecto Nuevo Acceso Sur a Santiago. Manuscrito en posesión del Consejo de Monumentos Nacionales.

Cornejo, L., F. Falabella y L. Sanhueza

2003-2004 Patrón de asentamiento y organización social de los grupos Aconcagua de la cuenca del Maipo. Revista Chilena de Antropología 17:77-104.
Dillehay, T.

1992 Identificación de grupos sociales y límites entre los mapuche de Chile: implicaciones para la arqueología. En Arqueología en América Latina Hoy, editado por G. Politis, pp. 144-156. Biblioteca Banco Popular, Bogotá.

1995 Mounds of social death: Araucanian funerary rites and political succession. En Tombs for the Living: Andean Mortuary Practices, editado por T. Dillehay, pp. 281-313. Dumbarton Oaks Research Library and Collection, Washington D.C.

Falabella, F.

2000 El sitio arqueológico de El Mercurio en el contexto de la problemática cultural del período alfarero temprano en Chile central. Arqueología de Chile Central (II Taller1994). (Abril 2005). http://www.arqueologia.cl/actas2/ falabella.pdf (15 diciembre 2005).

Falabella, F., E. Aspillaga, R. Morales, M.I. Dinator y F. Llona 1995-1996 Nuevos antecedentes sobre los sistemas culturales en Chile central sobre la base de análisis de composición de elementos. Revista Chilena de Antropología 13:29-60.

Falabella, F. y M.T. Planella

1979 Curso Inferior del Río Maipo: Evidencias Agroalfareras. Tesis para optar al grado de Licenciada en Arqueología y Título de Arqueóloga, Departamento de Antropología, Universidad de Chile, Santiago.

1991 Comparación de ocupaciones precerámicas y agroalfareras en el litoral de Chile central. Actas XI Congreso Nacional de Arqueología Chilena, Tomo 3, pp. 95-112. Museo Nacional de Historia Natural, Sociedad Chilena de Arqueología, Santiago.

Falabella, F., M.T. Planella, E. Aspillaga, L. Sanhueza y R. Tykot 2007 Dieta en sociedades alfareras de Chile central: aporte de análisis de isótopos estables. Chungara Revista de Antropología Chilena 39:5-27.

Falabella, F. y L. Sanhueza

2005-2006 Interpretaciones sobre la organización social de los grupos tempranos de Chile central: alcances y perspectivas. Revista Chilena de Antropología 18:105-134. 
Falabella, F. y R. Stehberg

1989 Los inicios del desarrollo agrícola y alfarero: zona central (300 a.C. a 900 d.C.). En Culturas de Chile. Prehistoria, editado por J. Hidalgo, V. Schiappacasse, H. Niemeyer, C. Aldunate e I. Solimano, pp. 295-311. Editorial Andrés Bello, Santiago.

Gaete, N.

1998 Estudio proyecto Rungue, Línea de base Patrimonio Cultural. Manuscrito en posesión del Consejo de Monumentos Nacionales.

Hagn, J.C.

1998 Sitios arqueológicos detectados en el sector de los cerros de Lonquén y zonas aledañas. Manuscrito en posesión del Consejo de Monumentos Nacionales.

Hermosilla, N.

1998 Proyecto relleno sanitario El Rutal. Montenegro, Comuna de Til Til, Región Metropolitana. Manuscrito en posesión del Consejo de Monumentos Nacionales.

2001 Informe de trabajos de ampliación de Línea de base arqueológica, sitio La Granja. Manuscrito en posesión del Consejo de Monumentos Nacionales.

Jochim, M.A.

1976 Hunter Gatherer Subsistence and Settlement: A Predictive Model. Academic Press, New York.

Johnson, A.

1989 Horticulturalists: Economic behavior in tribes. En Economic Anthropology, editado por S. Plattner, pp. 4977. Stanford University Press, California.

Johnson, A.W. y T. Earle

1987 The Evolution of Human Societies. From Foraging Group to Agrarian State. Stanford University Press, Stanford, California.

Massone, C.

1978 Cerro Blanco. Antropología de un Asentamiento Humano. Tesis para optar al Título de Antropólogo, Departamento de Antropología, Universidad de Chile, Santiago.

Ocampo, C.

1999 Sitio Metrogas calle Eduardo Acevedo Nº 2350-2380, Vitacura. Manuscrito en posesión del Consejo de Monumentos Nacionales.

Planella, M.T.

1988 La Propiedad Territorial Indígena en la Cuenca de Rancagua a Fines del Siglo XVI y Comienzos del XVII. Tesis para optar al grado de Magíster en Historia, Departamento de Historia, Universidad de Chile.

Planella, M.T. y F. Falabella

1987 Nuevas perspectivas en torno al período Alfarero Temprano en Chile central. Clava 3:43-110.

Planella, M.T. y B. Tagle

1998 El sitio agroalfarero temprano de La Granja: un aporte desde la perspectiva arqueobotánica. Publicación Ocasional del MNHN $\mathrm{N}^{\circ} 52$.

2004 Inicios de presencia de cultígenos en la zona central de Chile, períodos Arcaico y Agroalfarero Temprano. Chungara Revista de Antropología Chilena Volumen Especial Tomo I:387-399.

Quintanilla, V.

1983 Biogeografía. En Geografía de Chile Tomo 3. Instituto Geográfico Militar, Santiago.

Quiroz, L. y C. Belmar

2004 Estrategias de explotación de recursos vegetales: evidencia arqueobotánica de tres sitios de la región central de Chile: Radio Estación Naval, El Cebollar y Lonquén
(E 80-4). Chungara Revista de Antropología Chilena Volumen Especial Tomo II:1109-1119.

Rafferty, J.E.

1985 The archaeological record on sedentariness: Recognition, development, and implications. En Advances in Archaeological Method and Theory 8, editado por M.B. Schiffer, pp. 113-156. Academic Press, New York.

Rees, C.

2003 Informe EIA Gas Andes Pirque-Caletones. Manuscrito en posesión del Consejo de Monumentos Nacionales.

Reyes, V.

2001 Proyecto extensión Línea 5 Plaza Baquedano-Matucana (fase evaluación). Manuscrito en posesión del Consejo de Monumentos Nacionales.

2003 Proyecto extensión norte Línea 2 Cal y Canto-Cerro Blanco. Piques y Galerías. Manuscrito en posesión del Consejo de Monumentos Nacionales.

Salazar D. y R. Mera

1997 Informe de terreno arqueológico. Línea de base de recursos arqueológicos para el Proyecto inmobiliario Las Brisas de Chicureo, Comuna de Colina, Región Metropolitana. Manuscrito en posesión del Consejo de Monumentos Nacionales.

Sánchez R.

2001 Rescate arqueológico. Componente funerario sitio San José. Región Metropolitana, Provincia de Chacabuco, Comuna de Colina. Manuscrito en posesión del Consejo de Monumentos Nacionales.

Sanhueza, L.

2004 Estilos Tecnológicos e Identidades Sociales Durante el Período Alfarero Temprano en Chile Central: Una Mirada Desde la Alfarería, Tesis para optar al grado de Magíster en Arqueología, Departamento de Antropología, Universidad de Chile, Santiago.

Sanhueza, L. y F. Falabella

1999-2000 Las comunidades alfareras iniciales en Chile central. Revista Chilena de Antropología 15:29-47.

2006 Hacia una inferencia de las relaciones sociales del complejo Llolleo durante el período alfarero temprano en Chile central. En Procesos Sociales Prehispánicos en los Andes Meridionales, Universidad de Buenos Aires, en prensa.

Sanhueza, L., M. Vásquez y F. Falabella

2003 Las sociedades alfareras tempranas de la cuenca de Santiago. Chungara Revista de Antropología Chilena 35:23-50.

Seelenfreund, A.

1996 Restos arqueológicos asociados al Proyecto Inmobiliario Manso de Velasco, Comuna de Pudahuel, Región Metropolitana. Manuscrito en posesión del Consejo de Monumentos Nacionales.

1999a Informe de excavación de pozos de sondeo San Luis de Colina, Región Metropolitana. Manuscrito en posesión del Consejo de Monumentos Nacionales.

1999b Inspección arqueológica. Proyecto inmobiliario Valle Grande, Quilicura, Región Metropolitana. Manuscrito en posesión del Consejo de Monumentos Nacionales.

2000 Informe de excavación de pozos de sondeo en los sitios arqueológicos afectados por el proyecto inmobiliario Ayres de Colina. Manuscrito en posesión del Consejo de Monumentos Nacionales. 
Silva, J.

1964 Investigaciones arqueológicas en la costa de la zona central de Chile, una síntesis cronológica. Arqueología de Chile central y áreas vecinas. III Congreso Internacional de Arqueología Chilena, pp. 263-273. Sociedad Chilena de Arqueología, Santiago.

Stehberg, R.

1976 Un sitio habitacional alfarero temprano en el interior de Quinta Normal, Santiago, datado en 180 a.C. En Homenaje al Dr. Gustavo Le Paige, S.J., editado por H. NieTéllez, E. meyer, pp. 127-140. Universidad del Norte, Antofagasta.

2004 Evolución histórica de la población mapuche del reino de Chile. 1536-1810. Historia Indígena 8:101-26.

Thomas, C., A. Benavente y A. Durán

1980 Análisis crítico comparativo del cementerio Parque la Quintrala, La Reina. Revista Chilena de Antropología 3:41-56.

Thomas, C., A. Benavente, C. Massone, N. Gaete, R. Sánchez, G. Serracino, D. Jackson, C. Becker, J.C. Hagn, F. Constantinescu y R. Isaurieta

1990 Arqueología de la comuna de Lampa. Informe Proyecto Fondecyt $\mathrm{N}^{\circ} 124088$. Manuscrito en posesión de Fondecyt.

Varien, M.D.

1999 Sedentism and Mobility in a Social Landscape. The University of Arizona Press, Tucson.
Westfall, C.

1996 Proyecto gasoducto Gasandes. Extensión V Región. Manuscrito en posesión del Consejo de Monumentos Nacionales.

2000 Informe arqueológico. Prospección mediante pozos de sondeo sirio Sauce Oriente, comuna de Til Til, Región Metropolitana. Para proyecto Planta de Tratamiento de Aguas Servidas de Til Til, EMOS S.A. Manuscrito en posesión del Consejo de Monumentos Nacionales.

2001 Informe arqueológico. Prospección mediante pozos de sondeo. Proyecto Estacionamiento subterráneo Santa Lucía-José Miguel de La Barra, Comuna Santiago, Región Metropolitana. Manuscrito en posesión del Consejo de Monumentos Nacionales.

2003 Hallazgo de osamentas humanas, camino Jorge Alessandri s/n ${ }^{\circ}$, Parcela 6, Lote 26-2, Champa, Comuna de Paine. Manuscrito en posesión del Consejo de Monumentos Nacionales.

Winterhalder, B.

1981 Optimal foraging strategies and hunter research in anthropology: Theory and models. En Hunter-Gatherers Foraging Strategies: Ethnographic and Archaeological Analysis, editado por B. Winterhalder y E.A. Smith, pp. 13-35. University of Chicago Press, Chicago. 